\title{
Instrumentation in Cathodic Protection Systems: Field Survey
}

\author{
Noor H. Abdulwahab \\ Computer Engineering \\ University of Basra \\ Basra, Iraq
}

\author{
Ali A. Abed \\ SMIEEE, MACM \\ Computer Engineering \\ University of Basra \\ Basra, Iraq
}

\author{
Mohammed A. Jaber \\ Physics and Polymer Technology \\ Polymer research center \\ Basra, Iraq
}

\begin{abstract}
Most metals in nature are unstable and have the tendency to move its state to a more stable state in other words converted to its low energy oxides and this process called corrosion. As Corrosion, become one of the major problems and serious risks facing the oil industry that should be taken into account, since the first day of establishment of an industrial or oil plant. The cathodic protection (CP) system as the field experience has been proved is the most effective technique used in combination with coating for reducing corrosion process or eliminating it. This technique as the name suggests relied on making the part to be protected as the cathode of an electrochemical cell. This work highlights the most issues and problems that come with designing $\mathrm{CP}$ system such as (coating defect, interference, stray currents, etc.) with the use of the most common instruments used for inspection and testing cathodic protection system to ensure the system integrity and if it is working properly. Additionally, by the use of these instruments many variables and valuable parameters have been taken such as $(\mathrm{PH}$, Soil resistivity, Coating thickness, moisture content, etc.) which will be used later for assessing system performance. The purpose of this article is to supply information and a basic knowledge, which will assist the corrosion engineer in the installation, operation, and maintenance of cathodic protection systems in an effective way. The corrosion engineer will be skillful to use not only the common electronic millimeters but also very specialized instrumentation.
\end{abstract}

\section{Keywords}

Cathodic protection, Underground corrosion, impressed current CP system, sacrificial anode CP system, Wireless monitoring.

\section{INTRODUCTION}

The principal methods for reducing the rate of corrosion on the metal structures (pipelines, tanks, etc.) are coatings and cathodic protection (CP). Coating functions as the metal isolator from the surrounding environment, which forms a barrier that aims to make no contact with the ambient electrolyte. Although the coating is the primary effective method for reducing the current needed for protection, it has found that where applied there are always holes and defects formed during the process of transportation of a pipeline [1]. Another issue to be aware of is that CP system like any other electrical systems needs to be powered using current source, which, in CP system called anodes. Magnesium and zinc anodes have the high potential (more negative) in the galvanic series that makes it corrode or sacrificed to protect the metal below it in the series. Sometimes, sacrificed anodes become lossless in protecting long-distance pipelines because it requires large numbers of anodes to supply the required current. This becomes cost-effective, so another $\mathrm{CP}$ method called impressed current is required for applying external source, which is normally a rectifier that changes (AC) to (DC). Consequently, the latter method will lead to the problem of interference where the current flows in a way that is undesirable that will lead to accelerate corrosion of the foreign structures lies near the one being protected. With the availability of today's modern technology in the field of cathodic protection system, no company operating in pipelines field should accept the fact that pipelines corrode or system damage. The major portion of the paper will focus on explaining and simplifying the way of using most of these instruments. Also, a case study on a CP system using both sacrificial anode cahodic protection (SACP) and Impressed current cathodic protection (ICCP) methods which are designed on the basis of the data collected from the pre-design instruments (like soil resistivity, $\mathrm{PH}$, etc.) has been proved its effectiveness using Post-designed testing instruments (like holiday detector, DCVG, etc.). The importance of the early recognition and diagnosis of the corrosion problems mentioned before using a modern measurements instrument raises the rate for predicting the future system needs for Inspection, Maintenance, and Rehabilitation requirements. These instruments vary in types according to its uses from very complex ones that allow the recording of many tests under computer control such as (CIPS, DCVG, Holiday detector, etc.) or very simple such as (voltmeters, Ammeters, Earth resistivity tester, etc.) [2]. the aim in designing $\mathrm{CP}$ system and any electrical system is to get a maintenance-free system.

\section{RELATED WORK}

Over the past several years many research studies have been proved that $\mathrm{CP}$ system would suffer from an acceleration of corrosion due to different factors but the most effective is the presence of AC interference [3],[4]. Jin Shijiu and others develop portable detecting instruments that can detect the coating defect and coating disbondment simultaneously [5]. In 2016, Young-don Ryou, et al. [10], examined the measurement difficulties of the gas pipeline potential in the urban area where personnel attendant at drive roads specifically at the test station location is impossible due to the traffic at daytimes. So, they used two remote monitoring techniques, the first is an inspector car, they found it is unpractical to use this moveable technique due to the limitations in the transmission of data, traveling speed during testing process, and the distance which must be less than 20 meter in radius. In 2011, M. Hilmi and N. Hanis, [11], discussed the uniqueness property in using SMS based system among the remaining available techniques in monitoring $\mathrm{CP}$ system. As mobile phones nowadays become an integral part of our daily lives so, the accessibility at any time and from anywhere become easier by just sending a notify message 
with very low cost.

\section{ELICTRICAL EQUIPMENT}

The knowledge of instrumentation is essentially for anyone who wants to enter the field of cathodic protection. Hence, the life of a pipeline will be definitely infinite since all factors that cause corrosion has been revealed and controlled early. For this reason, field measurements has been taken for many parameters like (PH value, Soil resistivity, pipeline to soil potential, coating thickness, close interval potential (CIP), Direct Current Voltage Gradient (DCVG) survey, and Holiday detector all have been performed. This work involves two parts, the first one focus on how to use most of the modern instruments designed especially for testing the CP system. The second part represents a case study of a real system that has been designed by the reliance on the provided realistic parameters obtained from these instruments. Hence the results obtained has been proved how these instruments can provide a real problem solving that save time and money prior designing $\mathrm{CP}$ system.

\subsection{Voltmeters}

The first instrument that should be familiar with is the voltmeter. In a CP system, voltmeter used for the measurement of potential between the pipe that should be connected to the negative terminal of the meter and a copper sulfate reference electrode (CSE) which connected to the positive terminal that is called pipe-to-earth potential test that must be equal to -0.85 volt for buried pipelines as shown in Fig. 1. The potential reading on the meter display indicates the state of the pipeline if it is under protection, full protection or overprotection in other word using this instrument is to check if the criteria of complete $\mathrm{CP}$ is met. Therefore, the highimpedance, and high inner resistance compared with the circuit total resistance is necessary for avoiding misleading error. Additionally, meter reading can reveal fruitful information about the presence of high corrosion point or what called "hotspot region".

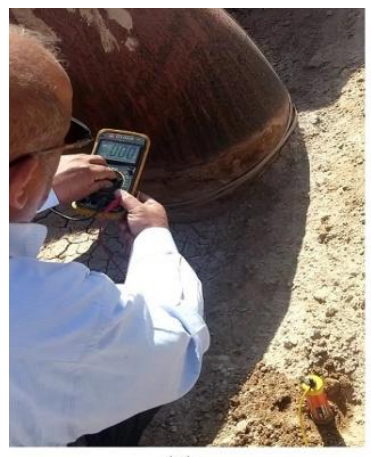

(a)

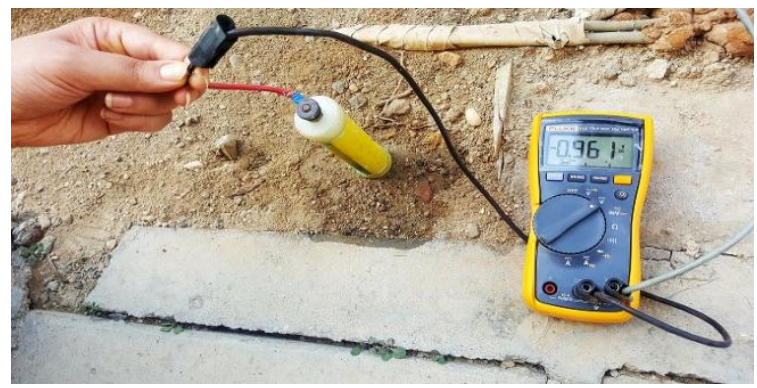

Figure 1 Handheld and Battery powered voltmeters

\subsection{PH meter and SD 320Con Conductivity Meter}

$\mathrm{PH}$ value has a great effect on corrosion indication and the needed current for the protection of the system [6]. This is done by different ways; the first chemical method is the use of the paper indicator. Usually, the paper is moistened with the liquid that is taken from the depth of the pipeline and the result is another different color, which is compared with color standards to indicate the approximate $\mathrm{PH}$ value. The second method is the use of an electrochemistry meters for more accurate result. In addition, the temperature has a direct effect on the criterion of complete cathodic protection of a pipeline, hence the change of polarization of the cathodes on the pipeline to the open circuit potential [6]. The displayed value has been recorded using the meter shown in Fig.2 (b) as 7.9, which indicate that the environment is alkali and has no effect on the CP system. In addition, The SD320 conductivity meter shown in Fig.2 (c) has been used to measure the conductivity of the water sample taken from the depth where the pipeline want to be buried. The value on the display screen has been recorded as $1503 \mathrm{mg} / \mathrm{l}$ for conductivity.

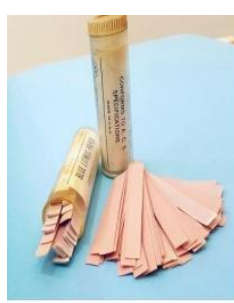

(a)

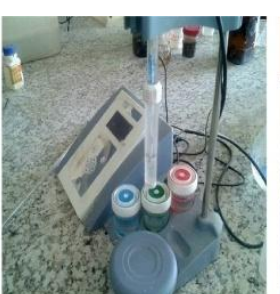

(b)

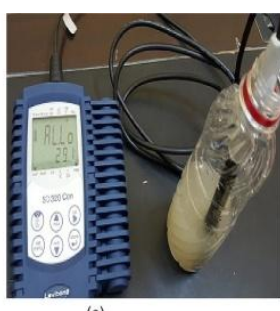

(c)
Figure 2 PH meters. (a): paper indicator, (b): Crison basic 20, (c): SD320con, and (d): measurement values.

\subsection{Soil resistivity meters}

Before listing instruments used in measuring soil resistivity, one may ask why to measure soil resistivity? On the other hand, what is the correlation between resistivity and controlling of pipeline corrosion? The answer is that resistivity has a direct impact on the degree of corrosion in an underground pipeline. A decrease in resistivity means increasing in corrosively. Another benefit in measuring resistivity in that it has a direct effect on the design of grounding system [7] because in order to reach the most amount of power it is important to locate the area of lowest soil resistivity. For this reason, focusing on the design of grounding system is the most important issue. The fourterminal method or Wenner method is the only method used here. In it, the average soil resistivity between the two center rods measured to a depth equal to the rod spacing. The average resistivity increases as the rod spacing increases, so at this depth, there is an area of higher soil resistivity, and if the average resistivity decreases with depth mean there is an area of lower soil resistivity. Fig. 3 shows the schematic of this method and the whole process shown in this video 


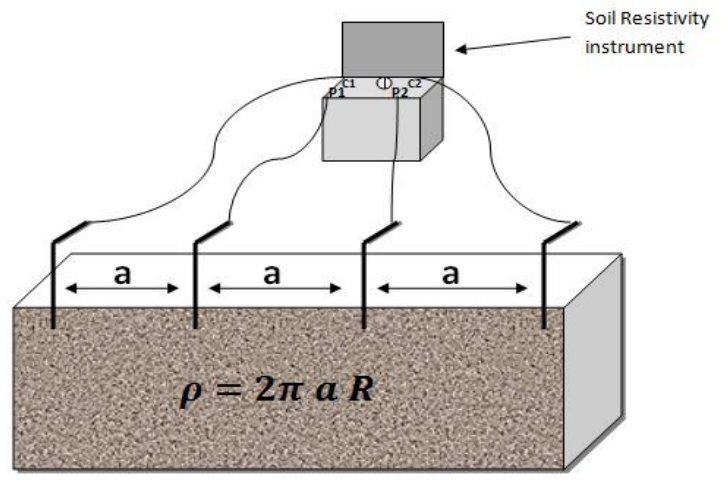

Figure 3 Wenner 4-pin method schematic

- Soil box

The soil box is the best and easiest method for measuring the soil or liquid resistivity of an electrolyte that removed from the environment for later calculations. The test results may sometimes be inaccurate because the natural moisture content of a soil sample cannot always be maintained. This involves the use of "four-pin Wenner method" so that Measurements can be taken and evaluated according to a pre known size of the box as shown in Fig.4 (d). This is the only disadvantage of using soil box that soil may dry and reading inaccurately taken but this can be handle by putting soil sample in plastic bags to keep soil moisture as it is.

- Nilsson model 400 4-pin soil resistance meter The meter shown in Fig.4 (b) can supply the required current to the exterior pins at the same time measuring dropping in voltage between the two center pins. Then computing the resistance, which is equal to the reading value shown on the dial selector multiplied by the position of range selector switch.

\section{- Hand-crank earth tester}

The main difference of this earth tester is to use the handle that is located on the right side of the device for ac generation instead of battery power as shown in Fig.4 (a). The meter functions by putting the position of the multiplier switch and resistance balancing dials to $\mathrm{x} 0.01$ and respectively. Then turning the handle two revolutions in one second at clockwise direction. Finally noticing the deflection on the meter, if the arrow goes to the right, it must increase the resistance factor by setting the switch position to $\mathrm{x} 0.1$, but if the arrow goes to the left, then decrease the dials one by one starting from the left to the right until noticing the balancing of the arrow on the meter.

\section{- $\quad$ DET4T2 megger tester}

The DET4T2 shown in Fig.4 (c) is a four-terminal digital instrument. It measures ground resistance from $0.01 \Omega$ to 20 $\mathrm{k} \Omega$ and earth voltages up to $100 \mathrm{~V}$. By using four rods driven down to equal depth and equal distances apart in a straight line. Four separate wires are connected to the rods and to the four terminals on the instrument. Hence, the name of this test: the four-terminal method. "Dr. Frank Wenner of the U.S. Bureau of Standards (now NIST) developed the theory behind this test in 1915". He showed that, the electrode depth (B) is kept small compared to the distance between the electrodes (A).

The following formula applies :

$$
\rho=2 \pi A R
$$

Where $(\rho)$ is the average, soil resistivity to a depth (A) in ohm-cm, (A) Is the distance between the electrodes in $\mathrm{cm}$, $\mathrm{B}=1 / 20 \mathrm{~A}$ is generally recommended, and $\mathrm{R}$ is the Megger earth tester reading in ohms.

Fig. 5 shows the reversely relationship between soil resistivity values at different distances where it can be noticed the increasing in distance yield to decreasing in resistivity.

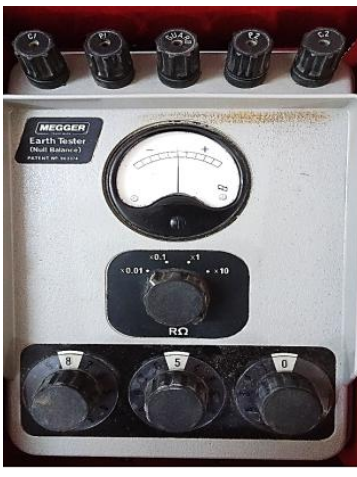

(a)

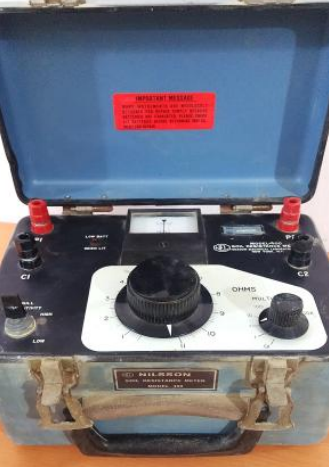

(b)

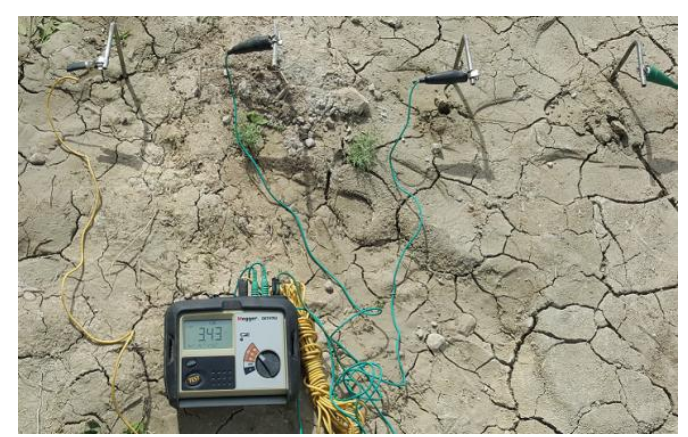

(c)

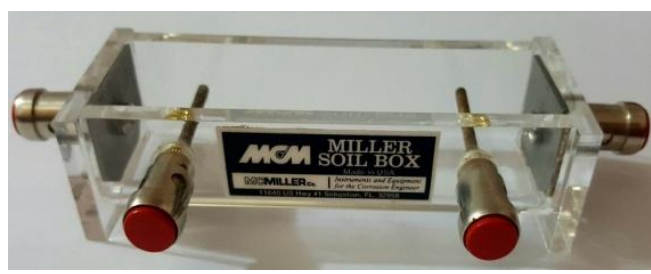

(d)
Figure 4 Soil resistivity meters. (a): Hand-crank earth tester, (b): Nilsson model 400,(c): Megger Soil Resistivity Tester and (d): Soil box

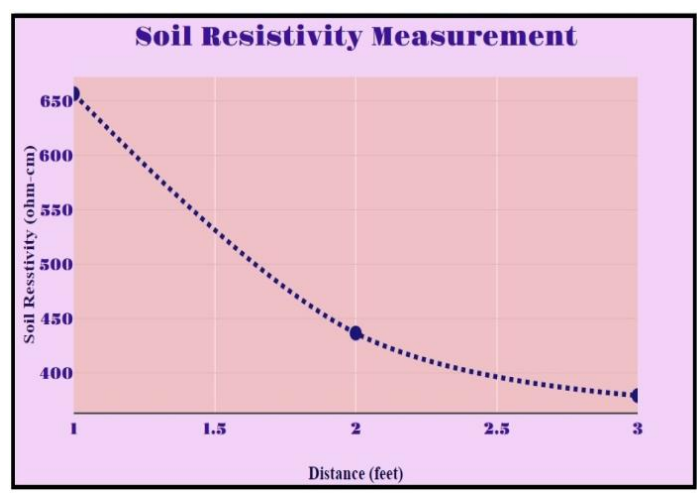

Figure 5 Measurement of Soil Resistivity at different distances. 


\subsection{Coating thickness gauges}

As shown in Fig.6 (a)(b) measuring the thickness of coating is very important since knowing the correct thickness ensures less current needed for protection and hence high system performance achieved. The gel that is shown in Fig. 6 (c) prepare an ideal connection by removing the air between the transducer and the material to be measured.

\section{- PosiTector 6000 Coating Thickness Gauge}

The positector shown in Fig.6 (a) is an electronic device that designed to be hold in the hand for an easier measurement taken with a fast and precise result estimating. It consists of the body of the gauge and probe. The main principle classified into three parts depending on its use: the first is the use of $F$ probes that is applicable to ferrous metals to measure nonmagnetic coatings. While the second probe called $\mathrm{N}$ probe and it is applicable to non-ferrous metals to measure the thickness of non-conductive coatings. At last using what called FN probes, which is the combination of the two $\mathrm{N}$ and $\mathrm{F}$ probes. The PosiTector 6000 supports interfacing with the computer for the assist in data viewing, analyzing, printing and reporting.

\section{- UNI-T UT342 Coating Thickness Gauge}

During the process of coating thickness measurement, it is sometimes necessary to hold a portable, lightweight gauge especially for a long pipeline to make the measuring process both quick and comfortable. Therefore, as shown in Fig.6 (b) the gauge is ideally designed for this purpose. The screen is placed on the handle to facilitate viewing results during usage. Furthermore, there are three buttons placed under the screen to facilitate access and control. Additionally, the device is capable of measuring paint thickness. ). Furthermore, the gel that is shown in Fig.6 (c) prepare an ideal connection by removing the air between the transducer and the material to be measured.

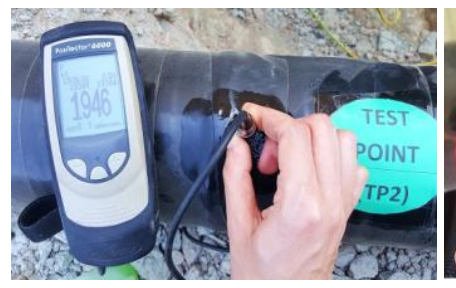

(a)

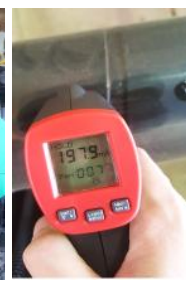

(b)

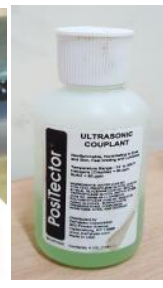

(c)
Figure 6 Coating Thickness Gauges. (a): PosiTector 6000, (b): Gel, and (c) UNI-T UT342

\subsection{Holiday detector}

While coating is considered as the main barrier protection technique and any flaws on it would not be visualized which lead to fasten the corrosion in the future if left without repair, it is necessary to ensure that it is perfectly applied for both new pipeline or buried one. Therefore, the process of continuous inspection and testing for coating is done by using a modern high voltage device called the coating holiday detector as shown in Fig.7 (a). The principle lies on supplying a high voltage to the pipeline using an electrode. This electrode takes many forms for the pipelines such as wire brushes such as the one shown in Fig.7 (b). When there is a defect, a spark takes place to indicate that there is a flaw in coating at this area, which should be specified and drawn by any marker for future repair. The inspector or surveyor must be familiar with the device to know the required voltage for use, which depends upon the quality of the coating. If the pipeline has a good coating quality there is no need to applying high voltages and reversely if it is poorly coated there is a high probability of holes existence and there is a need for a high voltage use. In addition, the thickness of coating play a role in voltage level indication, for pipelines the approximate voltage to use equal to $5 \mathrm{KV}$ in inspection a high degree of thickness like enamel coating [8]. In this work, first the grey curly cable has been connected to the instrument as shown in Fig.6 (b), then the clamp of the earth signal cable has been connected to a section of substrate as shown in Fig.7 (c). Finally, the applied voltage has been chosen as $10 \mathrm{KV}$, according to the coating thickness value that is measured previously according to ASTM G62-87 standard [14].

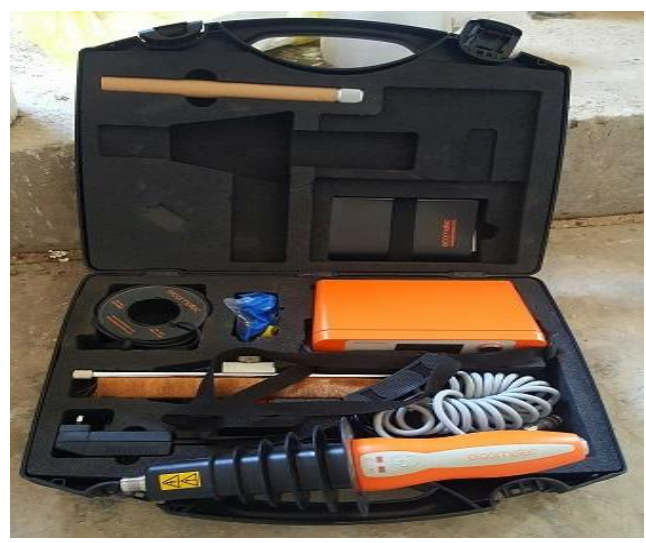

(a)

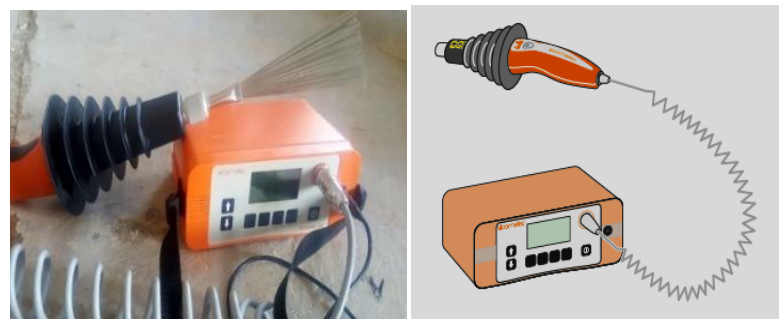

(b)

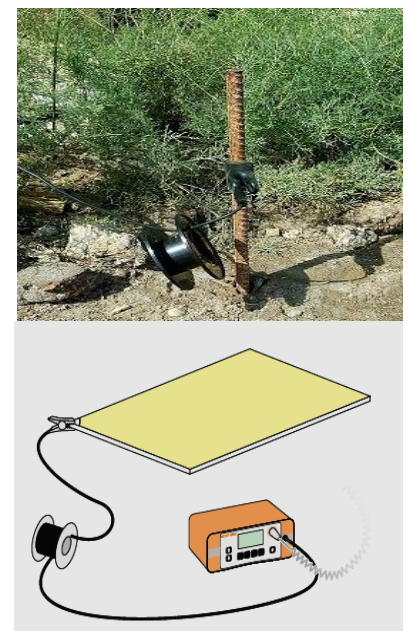

(c) 


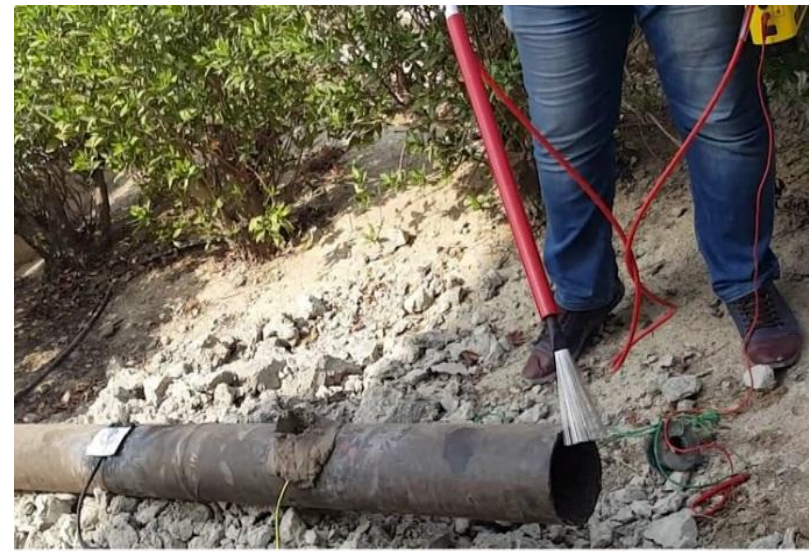

(d)

Figure 7 Holiday detector (a): The device package, (b): Wire brushes testing electrode, (c): Earth cable connection, and (d): Field survey tests.

\subsection{DCVG and CIPS}

Often there is a misunderstanding between CIPS and DCVG. CIPS is a technique for measuring pipe to earth potential, not as many thoughts as a coating defect locating, while DCVG is a coating flaw or defect locating technique not a pipe to soil

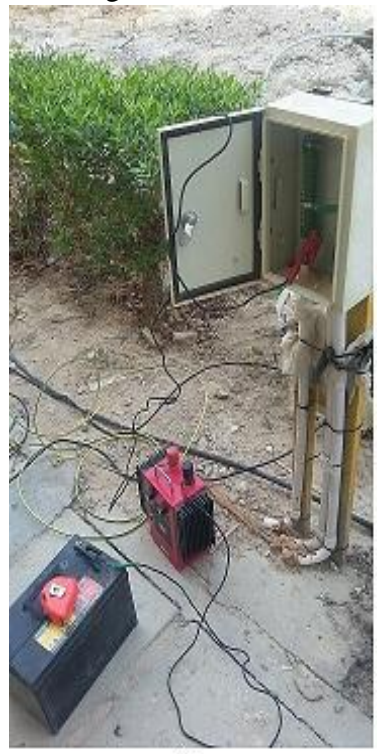

(a)

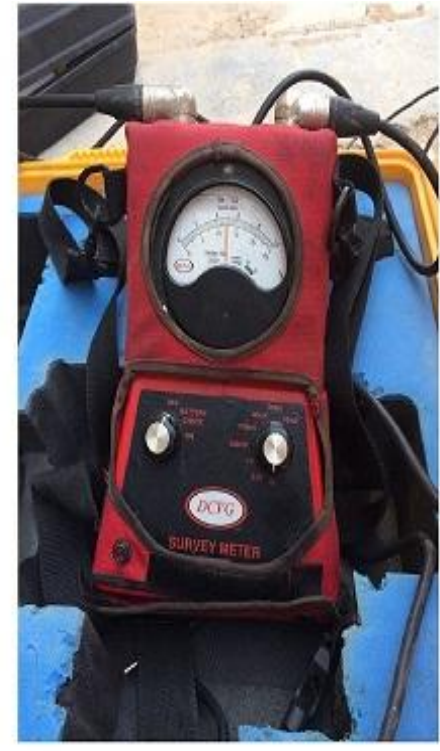

(b)

technique that is considered as a very precise measurement technique for locating a small severity defects [9]. New modern instruments combine the two techniques in one very powerful instrument representing the biggest step in the field of coating surveying for its easy and accurate measurements as shown in Fig.8. In the DCVG method, the indication of the difference in potential has been performed using two $\mathrm{Cu} / \mathrm{CuSo} 4$ reference cells that are placed at the earth where the structure being protected lies. The primary principle of this process involves pinpointing the direction of the current flow and the size of the potential gradient, which resulted from the comparisons of these two reference cells as one of them act as a more positive potential than the other did. The following video shows the two reference electrodes.

The interrupter shown in Fig.8 (a), which designed for pulsing the cathodic protection system on and off with a rating up to $50 \mathrm{Amp}$, has been connected to the buried pipeline. This enables the interruption (current output switching) at a rectifier or solar power station to remotely control. Using batteries or a portable DC generator as shown in Fig.8 (a) is necessary not only for DCVG technique but in most of the field surveys, that requires a rechargeable, and an easily replaced battery. The following video shows the whole process.

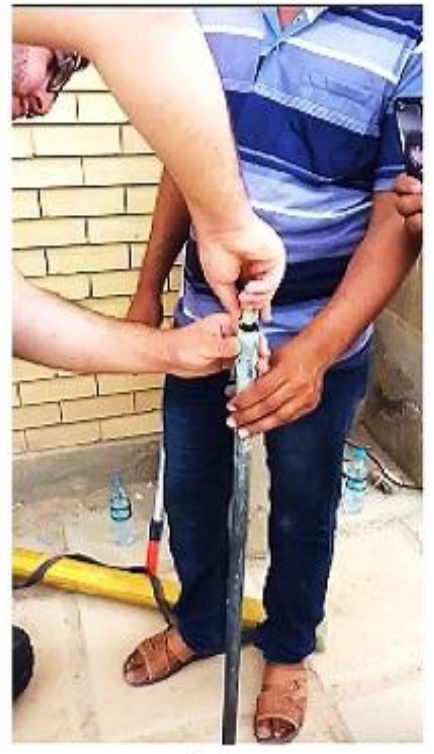

(c)

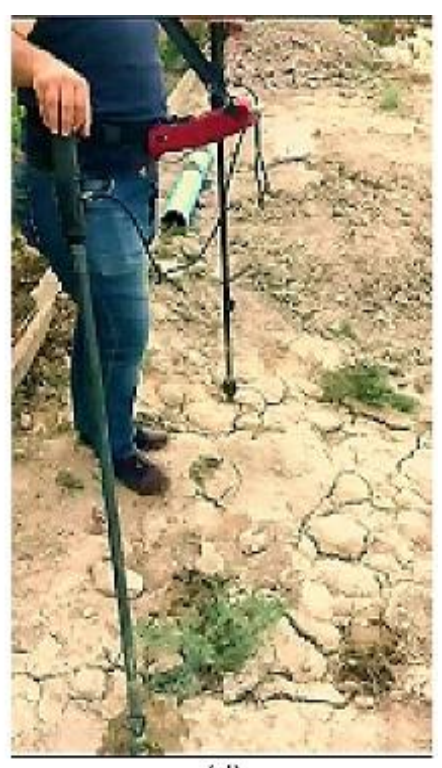

(d)

Figure 8 DCVG. (a): DCVG interrupter, and Battery, (b): DCVG meter, and (c), (d): DCVG probes

\subsection{Rectifier}

Rectifiers that are shown in Fig.9 are the most commonly used power source for impressed current cathodic protection systems. The most commonly used type of rectifier has an adjustable step down transformer, rectifying units (stacks), meters, circuit breakers, lightning arresters, current measuring shunts, and transformer adjusting points (taps), all in one case. When measuring current output of the transformer/rectifier (T/R) unit, it is preferred to use a shunt resistor to avoid disturbing the system as shown in Fig.9 (c). If the resistance known then the current output can be measured using the ohms law.

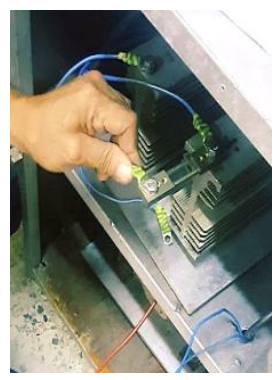

(a)

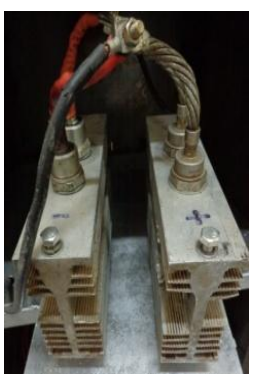

(b)

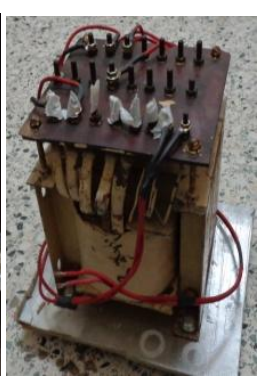

(c)
Figure 9 Transformer/Rectifier. (a): Rectifier, (b):Transformer, and (c): Shunt resistor. 


\section{- Terminal Connector box}

To connect the cables in a systematic and flexible way, the connecting box shown in Fig.10 is used. The Special Feature in this box is the existence of a writing bar in the middle of the box, which is intended to refer to the pipeline post points and anodes associated with it.

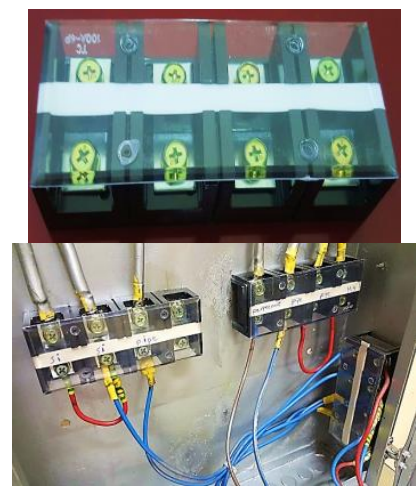

Figure 10 Terminal box

\section{- Amperage and voltage Analog Meters}

To display voltages and current values, the analog meters shown in Fig.11 have been used. Three gauges are mounted in the T/R panel. The first meter (which is placed on the left side) has been connected between the negative and positive terminals of the rectifier to display the voltage needed for full $\mathrm{CP}$ system protection. While the middle one has been connected to the terminals of shunt resistor to measure the protection current, and the last meter (which is placed on the right side of the panel) has been connected between the pipeline test point and the permanent reference electrode to be used as indicator of the pipeline to soil potential.

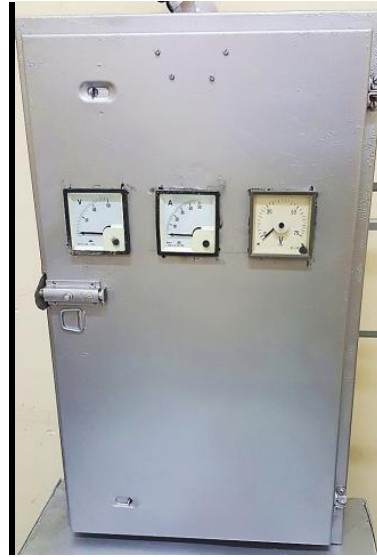

(a)

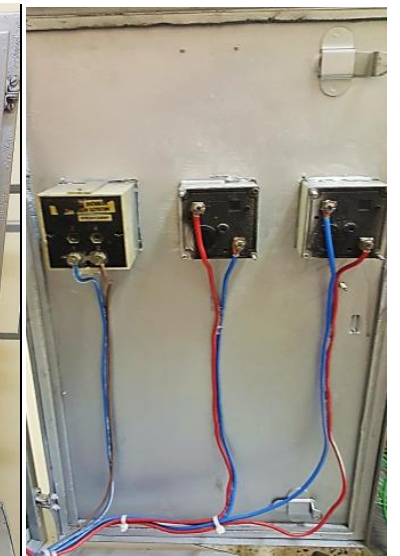

(b)
Figure 11 Amperage and voltage gauges. (a):The front side (b): The back side

\section{- Transformer/Rectifier Selector Switch}

It is well known that the transformer/rectifier unit converts the alternating current (AC) into a direct current (DC) and in several scales and ranges. So, the selector switch as shown in the Fig. 12 divided into 5 parts has been used to control the output voltages and select the range that provides the required protection to the pipeline.

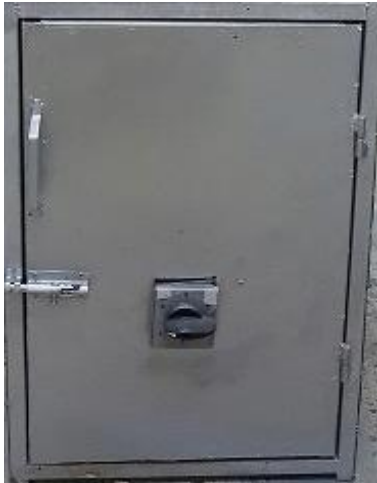

(a)

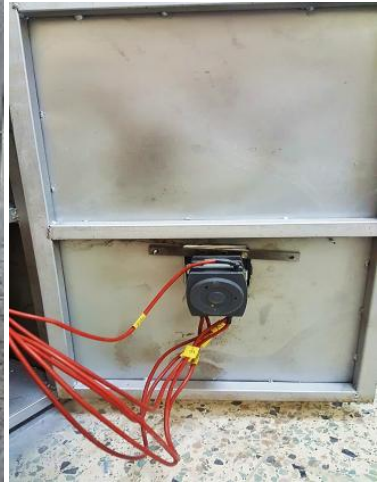

(9)
Figure 12 Selector Switch (a): The Front side (b): The back side

\section{- Junction box}

Most junction boxes are designed for transformer/rectifier (single-phase or three-phase input). All manufactured boxes are covered with a protective paint in order to resist against harsh conditions. In addition, the primary objective of these boxes are for monitoring (both manual and wireless), control, and test the pipe to soil potential and for resistance bonding to assure interference avoidance. The one that is used in this work as shown in Fig.13 is made and designed from scratch with very low-cost materials and has been implemented and proved to meet exact system requirements.

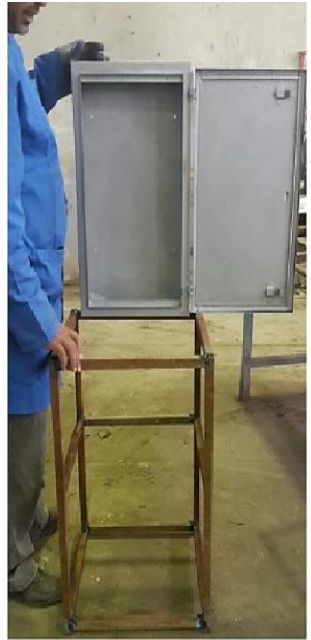

(a)

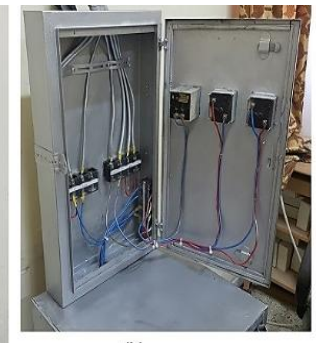

(b)

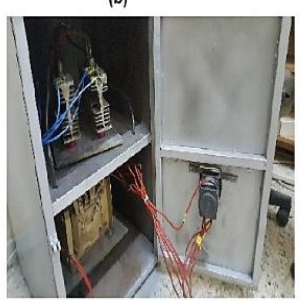

(c)
Figure 13 the transformer/rectifier panel

\subsection{Resistance bond}

The resistance Bond as shown in the Fig. 14 has been fixed between the structure to be protected and the foreign structure (specifically at the crossing point) to connect them to a $\mathrm{CP}$ system or to drain a stray current back to its source thus averting the interference problem. The value of this variable resistance, which is used in this work, is $1.4 \mathrm{ohms}$ (usually in the range of 2.5-to-3 ohm). The variable resistor have been adjusted by trial and error to make the foreign structures potential meet a steady value when measured with respect to $\mathrm{Cu} / \mathrm{CuSo} 4$ reference electrode (placed at the area of the structures intersection) in the case of turning the transformer/rectifier on and off or any current interrupter. 


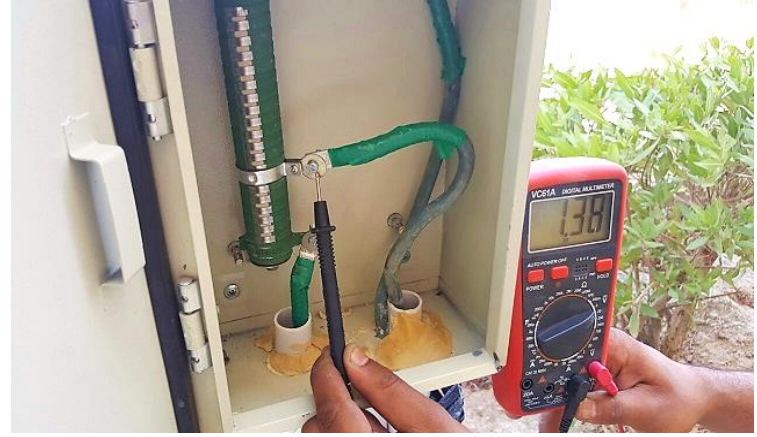

Figure 14 Variable bond resistance

\section{CONCLUSIONS}

The results of measuring different parameters using different instrumentation are presented. Firstly, the soil resistivity comparisons using three instruments have been applied and the average value has been selected. Secondly, the PH value has been estimated using the conventional paper indicator and modern instruments as 7.8, which indicates that the medium is alkaline. Third, the coating thickness has been measured using the most modern devices and the results showed that the value 1946 microns are enough to build a good defense barrier against corrosion. Forth, the coating holiday tests have been applied to make sure there is no holes or scratch in the coating that results from transportation process. In addition, the faults of coating have been located using the most widely used device, the DCVG. Finally, after data collection, the SACP and ICCP systems have been designed based on the survey from the previous measurements and the results showed that the pipe to soil potential ranges from 0.85 to 1.2 , which is the perfect protection due to the chosen of the low soil resistivity area, the coating tests applied, etc. Furthermore, the designed system has been monitored wirelessly using the GSM technique which specialized in the real-time monitoring, lowcost, and its availability anytime and from anywhere in the world.

\section{ACKNOWLEDGMENTS}

This work was done in the laboratories of the Faculty of Engineering in collaboration with the petrochemical company and the Basra Gas Company staff. Special thanks forward to the following people: Mr. Hasan A. Jasim the Manager of CP and chemical cleaning Unit of the petrochemical company,
Mr. Wael S. Abbood from Basra Gas Company, and Mr. Alaa Jasim. Finally, special thanks forward to the staff of polymer research center for their help and support in completing this work.

\section{REFERENCES}

[1] Underground pipeline corrosion, p.27 (2014)

[2] B. S. Wyatt: Underground pipeline corrosion. Elsevier Transl. vol. 2, pp. 2839-2856, (2010)

[3] L.Y. Xu, X. Su, Y.F. Cheng: Effect of alternating current on cathodic protection on pipelines, Elsevier, (2012)

[4] T. H. Shabangu, P.Shrivastava, and B. T. Abe, K. B. Adedeji and P. A. Olubambi: Influence of AC interference on the cathodic protection potentials of pipelines, IEEE Conference. Sept. (2017).

[5] Jin Shijiu, Li Jian, Chen Shili, Xiao Kun, Song Shizhe: The Study of Detection Technology and Instrument of Buried Pipeline-Coating Defaults", IEEE, (2002).

[6] Li Liangfu, Qin Binquan, Xiang Bo, and Yang Lei: Study on Impact of Precipitation PH and Conductivity on Soil Resistivity, IEEE, (2011)

[7] T. H. Shabangu, A. A. Ponnle, K. B. Adedeji K. B., B. T. Abe, P. A. Olumbambi and A. A. Jimoh "Effects of Soil Properties on Corrosion of Buried Steel Pipeline", IEEE, (2015)

[8] Sankara Papavinasam, "control in the oil and Gas Industry”, Elsevier, PP. 715-750(2014)

[9] Ramesh Sengh, Pipeline Integrity Handbook, PP. 3588(2017)

[10] Young-don Ryou, Jin-han Lee, Young-do Jo, Jin-jun Kim, Dong-kyun Kim: Development of a Remote Cathodic Protection Potential Measuring System Based on the IoT, Pipeline Technology Conference, (2016).

[11] Mohd Hilmi Hasan, and Nur Hanis Abdul Hamid, "Improving Cathodic Protection System using SMSbased Notification", International Journal of Computer Science and Information Security, Vol. 9, No. 2, 2011.

[12] UNI-T Model UT513 operating manual

[13]http://www.elcometer.com/en/coating-inspection/pinholeporosity/high-voltage-dc/elcometer-266-dc-holidaydetector.html

[14]https://www.spectro.com/products/mobile-metalanalyzer/spectroport-spectrometer 